\title{
Galactic Cepheid Kinematics as a Probe of Large Scale Non-Axisymmetry of the Galaxy
}

\author{
J. A. R. Caldwell ${ }^{1}$, C. Koen ${ }^{1}$, I. M. Avruch ${ }^{2}$, M. R. Metzger ${ }^{2}$, \\ P. L. Schechter ${ }^{2}$, M. J. Keane ${ }^{2}$
}

${ }^{1}$ South African Astronomical Obs., P.O.Box 9, Observatory 7935, S. Africa

${ }^{2}$ Massachusetts Inst. of Technology, Cambridge MA 02139 U.S.A.

${ }^{3}$ University of California, Santa Cruz CA 95064 U.S.A.

\begin{abstract}
We investigate whether the currently available Galactic Cepheid kinematic data can put interesting constraints on large scale low amplitude non-axisymmetry of the Galactic plane rotation pattern. In this connection we address the experimental design problem of where in the Galactic plane additional Cepheids would prove the most useful for the axisymmetric and the non-axisymmetric modeling of the kinematics.
\end{abstract}

\section{Optimal Design Selection of Galactic Cepheids to Constrain the Axisymmetric Rotation Model}

Caldwell and Coulson (1987) (hereafter CC87) analyzed the available photometry, reddenings, and radial velocities of Galactic Cepheids in terms of an axisymmetric Galactic rotation model, yielding $R_{0}$ relative to the Cepheid luminosity scale. Caldwell et al. (1992) (hereafter CAMSK92) reviewed the further observational progress over the intervening years, specifically the additional photometry and velocities, the improved reddenings, and the rapid relative increase of knowledge about distant Cepheids across the Galaxy. They redetermined the Cepheid rotation model with the new information, and derived $R_{0}=8.5 \pm 0.5 \mathrm{kpc}$, similarly to before, but with smaller uncertainty due to the accrued data. Two issues raised in that paper are the subject of further consideration here: the optimal choice of further Galactic Cepheids to search for and measure in future, and the possibility of large scale non-axisymmetry of the rotation pattern influencing the results.

The axisymmetric kinematic model fits the observed radial velocities of Galactic Cepheids by a 5-parameter function of the input variable, namely the Cepheid position in the Galactic plane. Four of the parameters appear linearly in the model (cf. CC87), namely the velocity shear $2 A R_{0}$, the solar motions $u_{0}$ and $v_{0}$, and the additive velocity zeropoint $\delta v_{r}$. The fifth parameter $m_{0}$ enters nonlinearly as a relative distance modulus scale. The problem of choosing where in the Galactic plane further Cepheids are to be detected and measured, in order to yield the most efficacious information about the parameters, comes under the statistics topic of optimal 


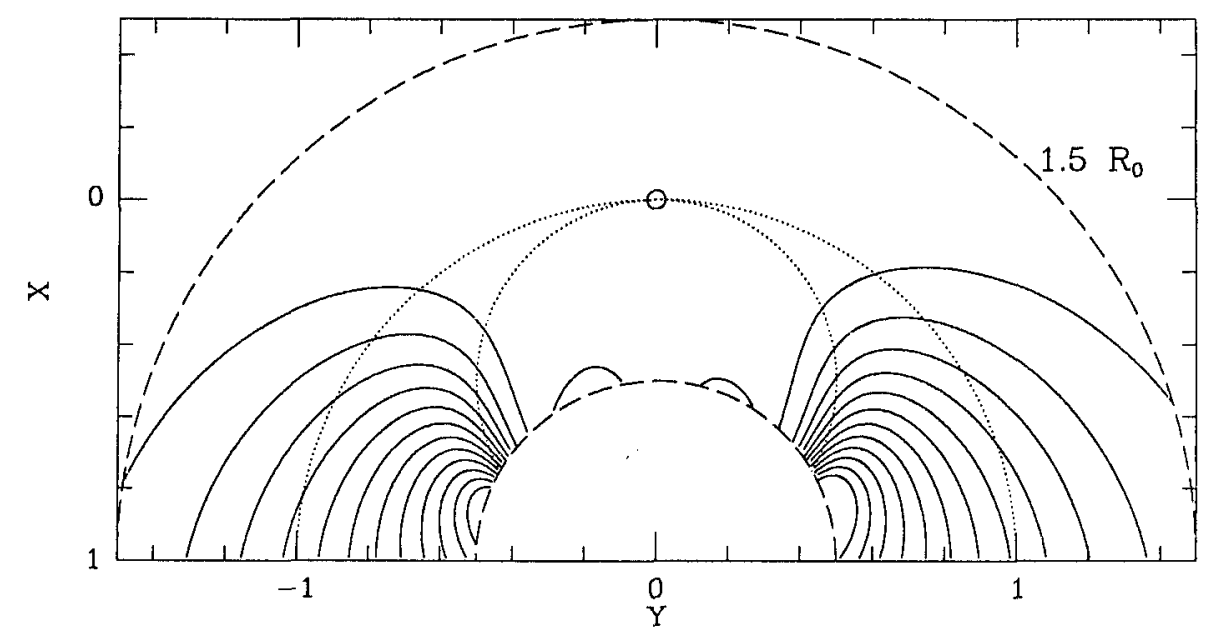

Fig. 1. The design parameter of the Cepheid axisymmetric kinematic problem. Increasing contours specify the desirability of obtaining new Cepheid data in an optimal design sense.

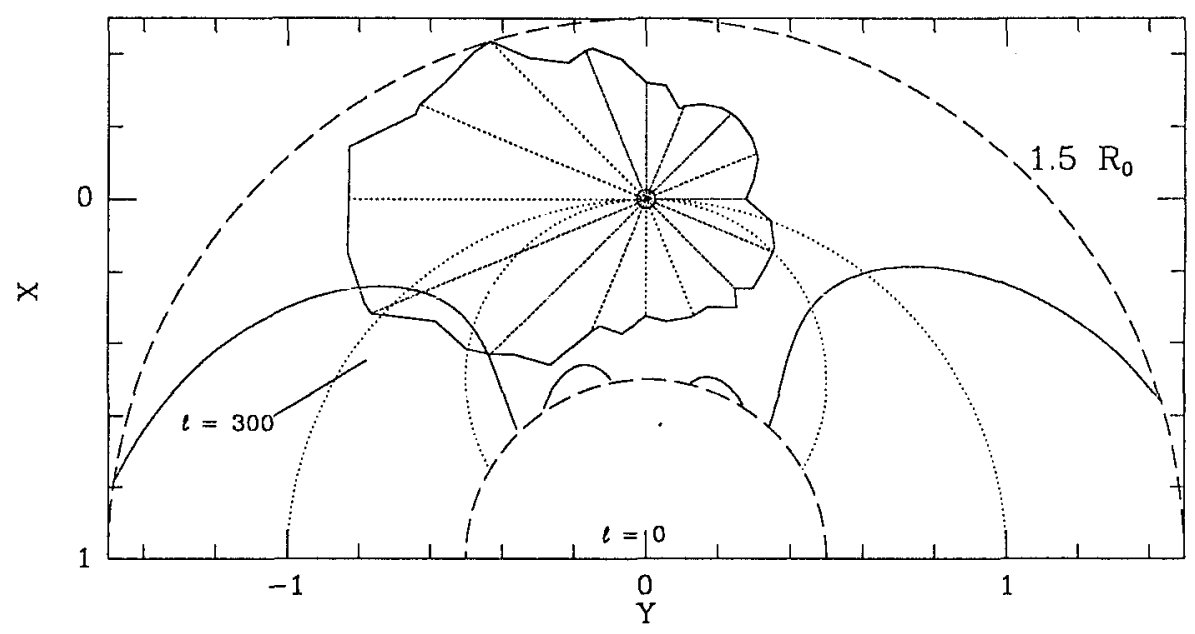

Fig. 2. The irregular outline encloses the region of the Galactic plane amenable to an analysis for Cepheid streaming velocities, with current data. 
design. Khuri and Cornell (1987, chap. 8) present a method for the optimal design of the values of the variables used to constrain a nonlinear model. In essence the design criterion is to maximize the design parameter, which is namely the $N \times N$ determinant of the variance-covariance matrix formed from the existing data plus one (or more) free design points, where $N$ is the number of model parameters. The maximization is carried out with respect to the design point(s).

Fig. 1 shows as solid lines the increasing contours of the design parameter, as a function of location on the Galactic plane. The design parameter, as remarked, indicates the relative efficacy of a new data point in maximizing the knowledge of the model parameters, as a function of where that new point is obtained. The origin at $(X, Y)=(0,0)$ is the position of the sun, and the solar circle and tangent point circle are shown as dotted lines. Fig. 1 confirms that new Cepheids brought to bear on axisymmetric kinematic problem are most useful to the extent that they breach the tangent point distance at longitudes from $30-70^{\circ}$ and $290-330^{\circ}$. An interesting detail of the figure is the north/south asymmetry of the design parameter, resulting from the imbalance in the currently available data (CAMSK92). Distant northern Cepheid data are now perceptibly more useful in constraining the model parameters than equivalently distant southern data. While at face value Fig. 1 gives high priority to new Cepheid data from deep chords across the Galaxy, one must bear in mind that the usefulness of such Cepheids depends critically on the assumption that the rotation curve is linear over the Galactocentric distance range concerned.

\section{Search for Large Scale Streaming Motions}

CAMSK92 presented the results of a kinematic model using a PLC relation (cf. CC87) to infer the Cepheid distances. We examine here whether the radial velocity residuals from the predictions of that model show any hint of "streaming" motions that could be the signature of large scale low amplitude non-axisymmetric distortion of the rotation pattern. Recent work especially by Blitz and Spergel (1991) and by Kuijken and Tremaine (1992) give the impetus to test the utility of Cepheids as tracers of such elliptical kinematic perturbations. We calculated a projected streaming velocity and projected distance for all Cepheid residual velocities from the axisymmetric solution prediction. This was done for a number of longitudes from $180^{\circ}$ to $337.5^{\circ}$ in steps of $22.5^{\circ}$, including for each direction only those Cepheids with a projection factor in absolute value within $10 \%$ of unity. The individual Cepheid velocities generally scatter with $\sim 11 \mathrm{~km} \mathrm{~s}^{-1}$ around the solution. To be more sensitive to possible streaming at the $5 \mathrm{~km} \mathrm{~s}^{-1}$ level, the points have been binned by 4 . Fig. 2 illustrates the direction vectors and heliocentric distance extent that the present binned data allow us to say anything about. Also shown is the lowest contour of design parameter discussed above. Clearly, as a handle on large scale non-axisymmetry at the few $\mathrm{km}$ $\mathrm{s}^{-1}$ level, the existing Cepheid data can offer only very limited information.

Fig. 3 shows the projected velocities in the specified longitude directions as a function of the projected distances in those directions. The only remarkable finding is a net contraction trend in $\ell=180^{\circ}$ direction and some possibly discrepant veloc- 
ities belonging to distant Cepheids towards $\ell=112-135^{\circ}$. The longitude $180^{\circ}$ trend resembles the differential velocity effect of an ellipsoidal distortion with the long axis pointing from the Galactic center into the first quadrant, and with radially decreasing amplitude. Otherwise there appears to be no possibly significant "streaming" trend in the data.

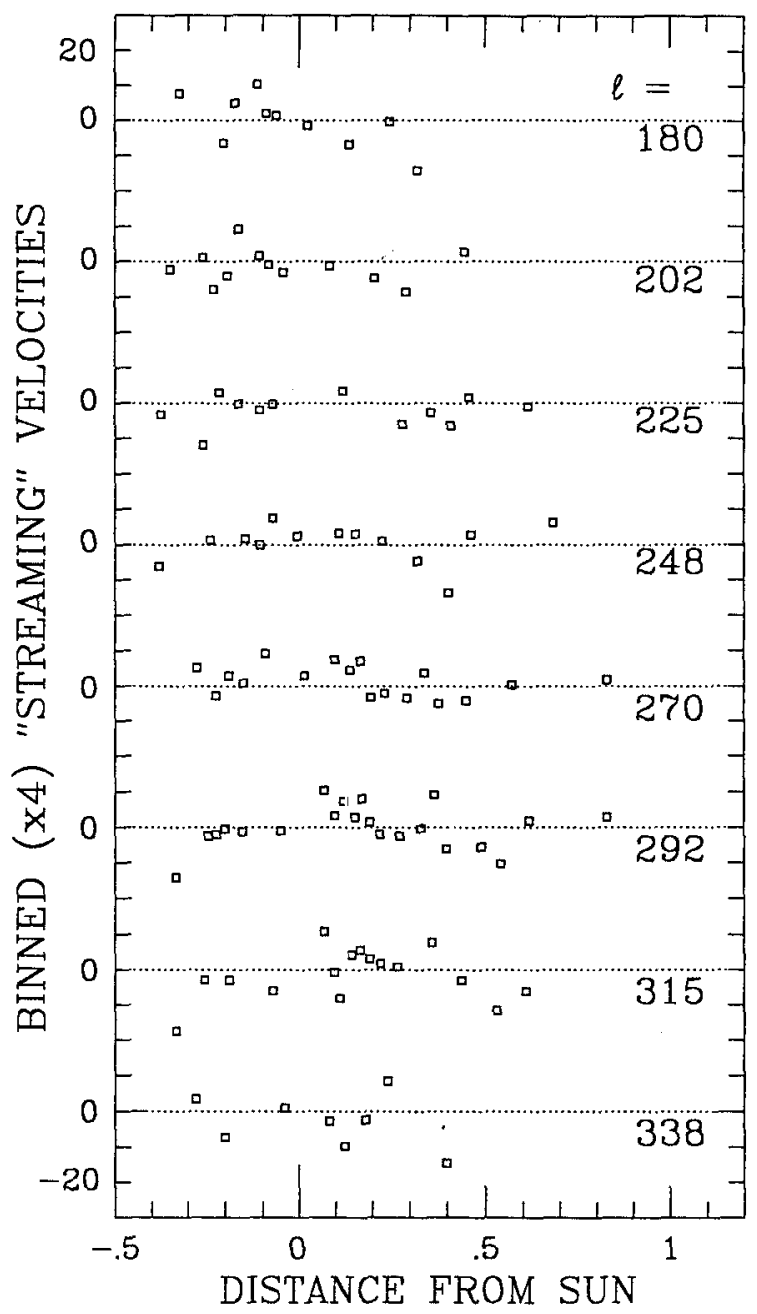

Fig. 3. Inspection of the velocity residuals from the axisymmetric kinematic solution, to look for "streaming" trends. 


\section{Non-Axisymmetric Simulation}

The program code used by CAMSK92 was expanded by the addition of a three-parameter ellipsoidal velocity perturbation. The ellipses are modeled by an axis-ratio-squared, $\kappa$, a tilt angle with respect to the anticenter vector, $\theta$, and a velocity normalization at the solar circle, $v_{p}$. The perturbation velocity magnitude is taken to behave in keplerian fashion with Galactocentric radius, and its direction is taken as following the elliptical streamlines. To keep the velocity perturbation more nearly differential, the purely circular velocity pattern corresponding to the same $\mathrm{v}_{p}$ is subtracted. The perturbed motion of the LSR is taken into account in computing perturbed radial velocities.

Fig. 4 illustrates the perturbation velocity pattern resulting from the model just described, with $\kappa$ and $\theta$ chosen to be $2^{\circ}$ and $-45^{\circ}$ respectively, that is with the long axis of the ellipses extending from the Galactic center into the first quadrant. This orientation is the same as adopted by Blitz \& Spergel (1991), and Whitelock (1992). The velocity contours indicate the relative perturbation radial velocity magnitude, and the signs are positive towards $\ell=90^{\circ}$ and $270^{\circ}$, and negative towards the center and anticenter. The hashed zones denote nearly zero net perturbation radial velocity. The expansion trend implied along the solar circle is if anything contradictory to the trend of the points in Fig. 3.

Fig. 5 shows the partial derivative of the radial velocity with respect to the principal other ellipsoidal parameter that one would ideally hope to be able to constrain by possible future Cepheid observations, $\theta$. Here the sign is again positive at the large peak in the first quadrant. It is apparent that one would need Cepheid velocities much farther across the Galaxy in this direction than are now available (cf. Fig. 2), to have good sensitivity to this parameter.

A number of nonlinear least squares solutions were attempted with the 8-parameter model (five from the axisymmetric case, plus $v_{p}, \kappa$, and $\theta$ ). The conclusions are that the present data do not constrain $\kappa$ and $\theta$. Further, when fixing $\kappa$ and $\theta$ at a variety of trial values, the radial velocities were best fit with no ellipsoidal perturbation added: $v_{p}$ converged toward zero in all attempts. Although this negative result was disappointing, but not surprising, plots such as Figs. 4 and 5 hold out the hope that the discovery of new Cepheids half way to the solar circle at $\ell=45-60^{\circ}$ will be directly useful in testing for large scale non-axisymmetry in future.

\section{References:}

Blitz, L., and Spergel, D. N. 1991, ApJ, 370, 205.

Caldwell, J. A. R., and Coulson, I. M. 1987, AJ, 93, 1090.

Caldwell, J. A. R., Avruch, I. M., Metzger, M. R., Schechter, P. L., and Keane, M. J. 1992, in: Variable Stars and Galaxies; symposium in honor of M. W. Feast, ASP Conference Series (in press).

Khuri, A. I., and Cornell, J. A. 1987, Response Surfaces: Designs and Analyses, Statistics: textbooks and monographs volume 81 .

Kuijken, K., and Tremaine, S. 1991, in: Dynamics of Disk Galaxies, 71.

Whitelock, P. A. 1992, in: Variable Stars and Galaxies; symposium in honor of M. W. Feast, ASP Conference Series (in press). 


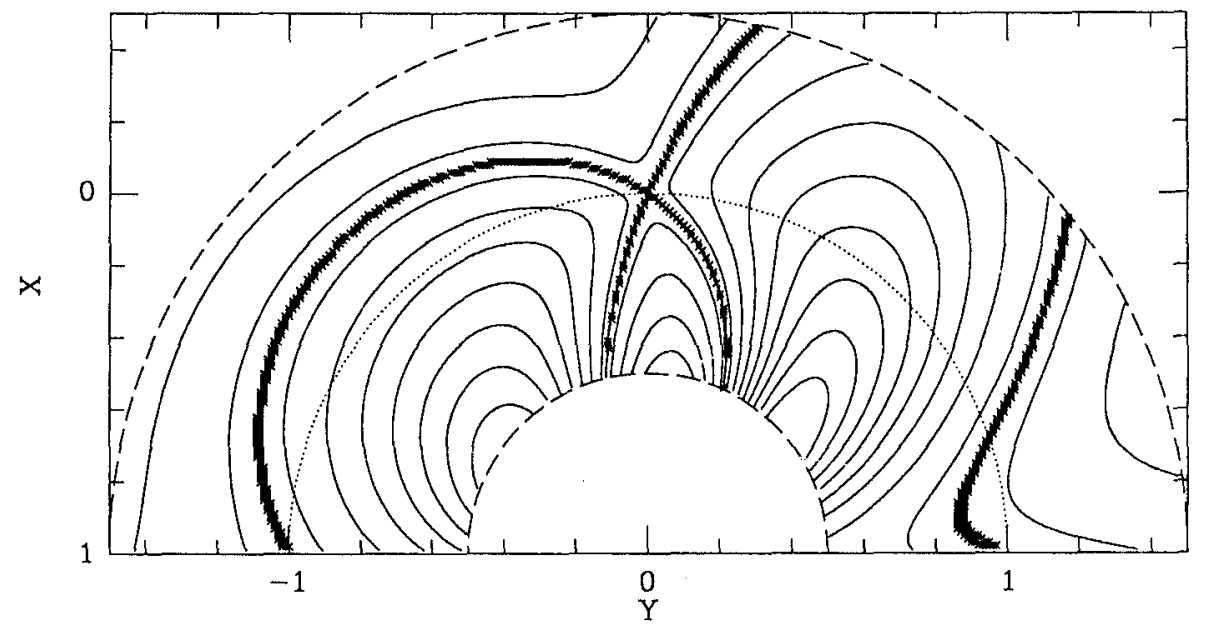

Fig. 4. Contours of the partial derivative of the observed Cepheid radial velocity with respect to the ellipsoidal perturbation velocity coefficient, $v_{p}$, as a function of Cepheid location in the Galactic plane, for a model discussed in the text.

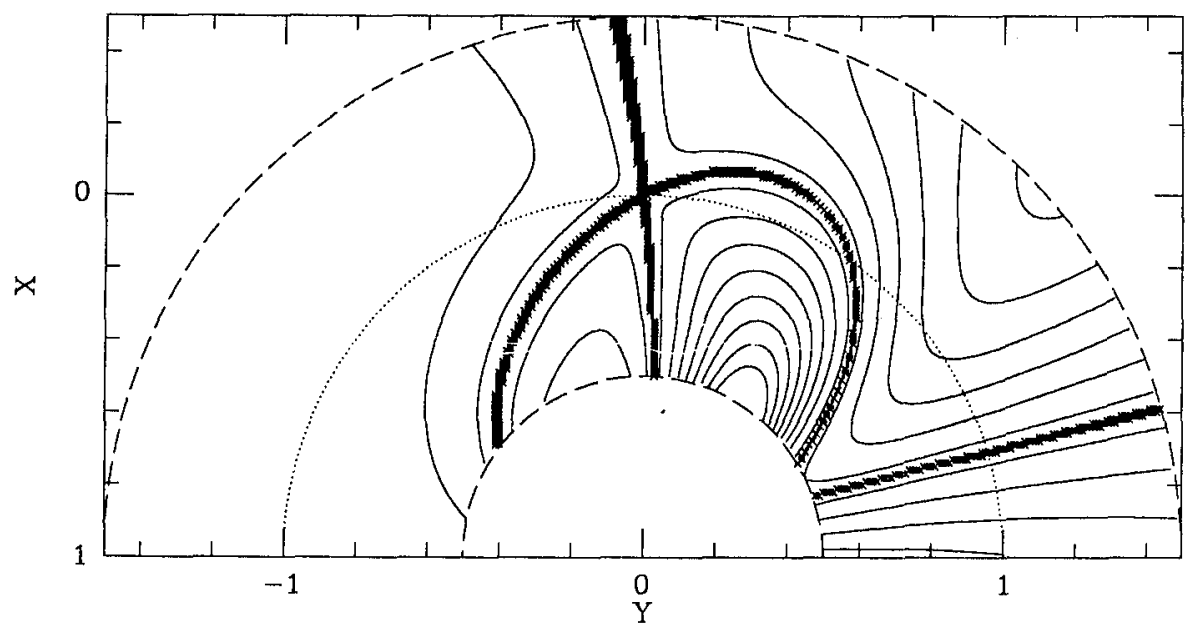

Fig. 5 Contours of the partial derivative of the observed Cepheid radial velocity with respect to the ellipsoidal perturbation tilt angle, $\theta$, as a function of Cepheid location in the Galactic plane, for the same model. 


\section{DISCUSSION}

D. TURNER: Is the solar motion relative to the LSR solved for in your analysis, and how does a spiral density wave affect your solution?

J. CALDWELL: Yes, the $u_{0}$ and $v_{0}$ velocities of the Sun are free parameters, that come out typically at 7 and $14 \mathrm{~km} \mathrm{~s}^{-1}$, respectively. I haven't yet looked into the effect of a spiral wave pattern. I suspect that there would be only a very minor effect because of the scaller in the Cepheid velocities and positions.

D. WELCH: I'm kind of surprised that you haven't mentioned outer disk Cepheids. Also, there is an asymmetry in the outer disk Cepheid velocities.

J. CALDWELL: The outer disk Cepheids will be extremely useful probes of the metallicity dependences of, e.g., the Cepheid $P L$ and $P C$ laws. Those towards $l \sim 135^{\circ}$ and $225^{\circ}$ strongly constrain $2 A R_{0}$. For the present though, I feel that additional distant solar circle or inner disk Cepheids would add the most to our understanding, for reasons I have presented previously. I will be very interested to know more about the asymmetry of the outer disk Cepheid velocities. 